\title{
La consultation du 7 décembre 2003 à la Martinique
}

Instrumentalisation politique et ambiguïtés conceptuelles

The December 7, 2003 Referendum in Martinique : political instrumentalisation and conceptual ambiguity

\section{Emmanuel Jos}

\section{OpenEdition}

\section{Journals}

Édition électronique

URL : http://journals.openedition.org/plc/66

DOI : $10.4000 /$ plc. 66

ISSN : 2117-5209

\section{Éditeur}

L'Harmattan

\section{Édition imprimée}

Date de publication : 15 décembre 2007

Pagination : $77-120$

ISBN : 2-296-01986-2

ISSN : 1279-8657

Référence électronique

Emmanuel Jos, "La consultation du 7 décembre 2003 à la Martinique », Pouvoirs dans la Caraïbe [En ligne], 15 | 2007, mis en ligne le 25 février 2011, consulté le 01 mai 2019. URL : http:// journals.openedition.org/plc/66 ; DOI : 10.4000/plc.66 


\title{
LA CONSULTATION DU 7 DECEMBRE 2003 A LA MARTINIQUE : INSTRUMENTALISATION POLITIQUE ET AMBIGUITES CONCEPTUELLES
}

\author{
Emmanuel JOS \\ Professeur de droit public \\ Membre du CRPLC \\ Université des Antilles et de la Guyane
}

\section{INTRODUCTION}

"Approuvez-vous le projet de création en Martinique d'une collectivité territoriale demeurant régie par l'article 73 de la Constitution et donc par le principe de l'identité législative avec possibilité d'adaptations, et se substituant au département et à la région dans les conditions prévues par cet article? ». Telle est la question figurant dans le décret présidentiel du 29 octobre 2003, et à laquelle les électeurs inscrits sur les listes électorales de la Martinique ont été invités à répondre par oui ou par non lors de la consultation du 7 décembre $2003^{1}$. Les électeurs n'ont donc été convoqués aux urnes que cinq semaines avant le scrutin, ce qui laissait peu de temps pour la campagne électorale.

Ce décret était introduit par deux visas, l'un faisant référence à la Constitution notamment son article 73, l'autre mentionnant «les délibérations du Congrès des élus départementaux et régionaux de la Martinique en date du 10 avril 2003, ensemble la lettre en date du 9 juillet 2003 par laquelle le président $d u$ Conseil général et le président du Conseil régional de la Martinique font connaître au ministre de l'outre-mer qu'ils approuvent le document d'orientation sur l'avenir institutionnel de la Martinique ».

Il faisait suite à une lettre adressée le 29 octobre 2003 par le Premier ministre Jean-Pierre Raffarin au Président de la République et rédigée dans les termes suivants : " J'ai l'honneur de vous proposer, au nom du gouvernement, qui en a délibéré lors du conseil des ministres du 29 octobre 2003, de consulter sur le fondement des articles 72-4 et 73 de la Constitution les électeurs de la Martinique sur le projet de création en Martinique d'une collectivité territoriale demeurant régie par l'article 73 de la Constitution et se substituant au département et à la région $»^{2}$.

\footnotetext{
${ }^{1} J . O \mathrm{n}^{\circ} 252$ du 30 octobre 2003, p. 18535.

${ }^{2}$ Ibidem.
} 
Un décret du 4 novembre 2003 est venu préciser l'organisation de la consultation des électeurs de la Martinique ${ }^{3}$.

Une déclaration du Gouvernement devant le Sénat et l'Assemblée nationale, suivie d'un débat, a eu lieu le 7 novembre 2003, soit un mois avant le scrutin populaire.

La campagne officielle a été ouverte le 24 novembre 2003 à zéro heure et close le 5 décembre à minuit. Elle n'a donc duré que douze jours. Ont été habilités à participer à la campagne, à leur demande, les partis et groupements politiques auxquels ont déclaré se rattacher trois élus au moins parmi les parlementaires, les conseillers généraux et les conseillers régionaux élus en Martinique.

Le jour du scrutin, les électeurs ont eu à leur disposition deux bulletins de vote l'un portant la réponse OUI et l'autre la réponse NON. Ils ont reçu les professions de foi des groupements habilités à faire campagne officiellement.

Le droit de vote était ouvert aux électeurs de nationalité française inscrits sur les listes électorales de la Martinique.

Le résultat du scrutin a donné une faible majorité au NON soit: $54695(50.47 \%)$ contre $53675(49.53 \%)$ pour le OUI. L'écart fut donc de 1020 voix $(0.94 \%)$. Le taux de participation a été de $43.92 \%$ (266 130 inscrits et 116896 votants). Il y a eu 8526 bulletins blancs ou nuls.

Ont voté majoritairement OUI les communes de Grand-Rivière, Prêcheur, Morne-Rouge, Schoelcher, Fort de France, Lamentin, Trois-Ilets, Anses-d'Arlet, Diamant, Rivière-Pilote et Sainte-Anne. Soit 11 communes sur 34. Parmi les villes ayant le plus grand nombre d'inscrits ont voté OUI : Fort de France, Lamentin, Rivière Pilote et Schoelcher. Ont voté majoritairement NON les communes suivantes: François, Robert, SaintJoseph et Sainte-Marie. Les écarts les plus grands numériquement en faveur du OUI ont été enregistrés à Fort de France (2422 OUI de plus que de NON) à Rivière Pilote (1674 OUI de plus que de NON) et au Morne Rouge (705 OUI de plus que de NON). Les écarts les plus grands numériquement en faveur du NON ont été atteints à Sainte Marie (1273 NON de plus que le OUI) et au Robert (1108 NON de plus que le OUI). Les bulletins blancs ou nuls les plus nombreux ont été recensés, par ordre décroissant, dans les

${ }^{3} J . O \mathrm{n}^{\circ} 256 \mathrm{du} 5$ novembre 2003, p. 18862. 
communes suivantes : Fort de France (1801), Sainte Marie (660), François (617), Lamentin (609) et Robert (570).

De nombreux enseignements peuvent être tirés de l'analyse de l'avant consultation et des résultats du scrutin. L'une des questions qui nous semble parmi les plus importantes soulevées par la consultation du 7 décembre est celle de savoir si les électeurs se sont véritablement prononcés en toute clarté. Certes, le Conseil d'Etat dans un arrêt du 4 décembre $2003^{4}$ a estimé qu'il n'y avait pas d'arguments juridiques fondés pour contester la loyauté et la clarté de la question posée par le Président de la République aux électeurs. En revanche, force est de constater que le déroulement de la campagne a été marqué par une instrumentalisation du droit telle qu'à mesure que l'on s'approchait de la consultation la confusion s'accroissait dans bon nombre d'esprits, en dépit des efforts pédagogiques entrepris. Cette instrumentalisation, il faut le reconnaître, a été en grande partie facilitée par l'ambiguité des textes et les divergences d'interprétations doctrinales. Les interprétations opposées sur le point de savoir si la réforme proposée consistait en une évolution institutionnelle ou un changement de statut constituent une parfaite illustration des difficultés conceptuelles rencontrées à l'occasion de la consultation.

En effet, certains l'ont inscrite dans la problématique de l'évolution institutionnelle et d'autres l'ont contestée en estimant qu'il s'agissait d'un changement de statut et en mettant en avant l'indétermination du contenu de ce statut.

Au-delà de l'instrumentalisation politique, la controverse a eu le mérite de soulever un certain nombre de questions juridiques au nombre desquelles il y a notamment celles-ci : que faut-il entendre par statut et par évolution institutionnelle lorsqu'il s'agit de collectivités situées outre-mer? Le régime de l'article 73, issu de la révision du 28 mars 2003, est-il encore celui de l'identité législative avec adaptation ?

\footnotetext{
${ }^{4}$ CE, 4 décembre 2003, M. Feler, req. N²62009, concl. Mitjavile, rapp. Melle Herry.
} 


\section{Au-delà de l'instrumentalisation politique : les notions de statut et d'évolution institutionnelle des collectivités territoriales situées outre-mer}

L'instrumentalisation politique de la notion de statut départemental a consisté à attiser les peurs latentes dans la conscience de nombre d'électeurs martiniquais en donnant à croire que les avantages sociaux étaient liés au maintien de la collectivité départementale. Le terrain était particulièrement favorable compte tenu d'une histoire qui a inscrit profondément dans la conscience collective martiniquaise que l'intégration dans la République française et la possibilité de bénéficier des avantages sociaux étaient liées intrinsèquement à la structure départementale. Cette erreur, communément répandue et volontairement entretenue, était d'autant plus difficile à dissiper que, parallèlement, la réflexion juridique faisait apparaître des hésitations et des divergences sur la signification des notions de statut, de statut particulier, et d'évolution institutionnelle.

\section{A - L'attisement des peurs}

L'exemple le plus caricatural de l'attisement des peurs avant la consultation du 7 décembre 2003 a résidé dans la distribution, en de très nombreux exemplaires, d'un tract libellé comme suit :

«ON

Le 7 décembre 2003 sans hésitation

Il faut voter NON

NON à l'indépendance

NON à la perte de notre département et de notre région

NON à la perte de nos acquis sociaux

NON à la perte du RMI

NON à la perte des $40 \%$ des fonctionnaires

NON à la perte de notre pouvoir d'achat

NON au retour du colonialisme dans notre île 
NON au pouvoir unique et dictatorial

NON au chant des sirènes

NON à l'avenir dangereux pour nos enfants

NON à des impôts supplémentaires

PAS ACHETER CHAT' DAN SAK ${ }^{5}$ ».

Ce tract utilisait au service du NON l'agitation des peurs en donnant à la réforme proposée une signification inverse de ce qu'elle proposait. Il n'était pas question d'indépendance et l'option retenue, par les initiateurs de la réforme, pour la nouvelle collectivité était qu'elle demeure sous le régime de l'article 73, autrement dit de l'application de plein droit des lois et règlements. Le tract, par un amalgame délibéré, laissait entendre que la pérennisation des avantages acquis était liée au maintien des collectivités départementale et régionale, alors qu'elle dépend d'une part du régime législatif de l'application de plein droit et d'autre part de la volonté politique des gouvernants. S'agissant de l'importance de la volonté politique, il est clair, pour ne retenir que cet exemple, que le maintien des $40 \%$ de vie chère en plus du traitement des fonctionnaires ne résulte pas du régime législatif. Il dépend en réalité d'une décision politique et des rapports de force sociaux.

L'instrumentalisation politique se retrouve dans le texte de la page entière payée par des partisans du NON dans le quotidien local FranceAntilles du 27 octobre 2003 :

«Le 7 décembre prochain, les Martiniquaises et les Martiniquais sont invités à se prononcer sur le projet d'évolution statutaire qui est proposé.

Ceux qui présentent ce projet comme un moyen de simplification administrative nous trompent car ils nous cachent l'essentiel, à savoir: les conséquences qu'entraîne la suppression du département et de la région, pour les remplacer par une collectivité nouvelle aux conséquences inconnues:

\section{Mi sac la !!}

\footnotetext{
${ }^{5}$ N'achetez pas de chat dans un sac.
} 
Ces compétences seront définies après le 7 décembre par une mystérieuse loi organique : mi chatt la !!

Ce rendez-vous est décisif pour l'avenir de la Martinique car la suppression du département et de la région met en péril nos droits actuels et tous les droits que nous pourrions obtenir en conservant à la Martinique sa qualité de département français.

L'heure est donc venue de nous mobiliser pour faire obstacle au projet de collectivité nouvelle des autonomistes et indépendantistes.

Il appartient à chaque martiniquais et à chaque martiniquaise de prendre toute sa part dans cette mobilisation vitale et ce combat décisif.

Il faut donc voter NON au projet dangereux de suppression du département et de la région.

Moun Matinik pa ka acheté chatt dans sac !! »

Ce texte, qui s'apparente à un tract, émanait d'un groupe intitulé : «Pour la Martinique Département Français ». L'argument est ici tout à fait clair: les droits actuels et futurs sont liés à l'existence de la collectivité départementale. On note également dans ce texte une erreur savamment distillée tout au long de la campagne par les adversaires de la réforme à savoir que la création, l'organisation et les compétences de la collectivité nouvelle relevaient d'une loi organique. La vérité est que la création de la collectivité territoriale nouvelle et la détermination de son organisation ainsi que de ses compétences relevaient sans aucun doute possible de la loi ordinaire $^{6}$. La référence à la loi organique entretenait l'amalgame avec le passage à l'article 74. Dans ce cas, en effet, la collectivité nouvelle régie par l'article 74 , et non par l'article 73 , voyait notamment sa création, le contenu de son régime législatif, son organisation et ses compétences établis par une loi organique. La loi organique, considérée donc à tort comme instrument de création de la collectivité unique régie par l'article 73 , se trouvait ainsi instrumentalisée par les adversaires de la réforme en devenant un véritable épouvantail, alors que, dans le régime de l'article 73 , la loi organique est prévue pour préciser les procédures de demande d'habilitation, d'adoption

${ }^{6}$ L'article 73 alinéa 7 dispose : « la création par la loi d'une collectivité se substituant à un département et à une région d'outre-mer... » (souligné par nous). 
des actes pris en application des habilitations et les limites des pouvoirs nouveaux conférés par le texte de la Constitution révisée aux collectivités départementales et régionales situées outre mer et, en conséquence, également aux collectivités nouvelles qui seraient susceptibles de se substituer à elles. Cette loi organique ne concerne donc pas spécifiquement la collectivité nouvelle mais bien en premier lieu les collectivités départementales et régionales situées outre-mer. La preuve en est que la substitution d'une collectivité nouvelle aux collectivités existantes n'ayant pas eu lieu, la loi organique prévue est actuellement en préparation, les assemblées départementales et régionales situées outre mer ayant été consultées sur un projet de texte courant 2005.

Il convient de noter enfin à propos du texte précité, paru dans le journal France-Antilles du 27 octobre 2003, que la réforme est présentée comme un projet des autonomistes et indépendantistes alors qu'il était issu d'un compromis entre différentes tendances politiques, y compris des tendances politiques opposées à l'autonomie et à l'indépendance, et qu'il s'inscrivait dans un cadre qui, aux dires du gouvernement lui-même, avait été « verrouillé » pour éviter les évolutions non désirées par la majorité des électeurs. En effet, tout changement de régime est subordonné au consentement des électeurs concernés, en vertu de l'article 72-4 alinéa premier. Quant à l'accession à l'indépendance, le Conseil constitutionnel considère que les dispositions de l'article 53 alinéa trois prévoyant le consentement des populations intéressées "doivent être interprétées comme étant applicables dans l'hypothèse où un territoire cesserait d'appartenir à la République pour constituer un Etat indépendant ou y être rattaché ${ }^{7}$.

Dans une autre parution du quotidien local France-Antilles, la réforme était présentée comme devant engendrer une catastrophe du même ordre que la destruction de Saint-Pierre par la montagne Pelée en $1902^{8}$. L'appel à l'inconscient collectif et aux peurs qui y sont enfouies donne à réfléchir...

\footnotetext{
${ }^{7}$ Décision n ${ }^{\circ} 75-59$ DC du 30 décembre 1975, JO du 3 janvier 1976, p. 182.

${ }^{8}$ France-Antilles, 9 novembre 2003, p. 9.
} 


\section{B - Le poids de l'histoire}

L'amalgame entre appartenance à la République française, maintien des avantages sociaux et collectivité départementale était d'autant plus facile à entretenir qu'il est le résultat d'une problématique historique et d'habitudes de vocabulaire si profondément ancrées dans les esprits qu'il faudrait une véritable révolution culturelle pour qu'elles puissent changer afin d'entrer en adéquation avec les évolutions juridiques.

Comme l'indique François Miclo, ce qui caractérise la législation appliquée dans les colonies françaises à partir de 1789 c'est une oscillation entre "l'assimilation complète avec celle de la métropole, et le particularisme colonial. Elle s'explique par la tentative constante de concilier deux exigences: l'une juridique qui entend faire en sorte que les colonies soient sous la souveraineté française comme les autres régions métropolitaines; l'autre de nature géographique et économique qui nécessite des particularismes.

La conciliation s'avère difficile à réaliser: le plus souvent l'un des deux l'emporte sur l'autre »".

Dans les périodes où la tendance assimilationniste a dominé, la démarche consistait à affirmer premièrement le principe de l'intégration des colonies dans la République pour ensuite en tirer comme conséquence la mise en place dans ces colonies de la même organisation administrative qu'en métropole, en l'occurrence la division en départements. C'est ce qui a été fait par exemple par la Constitution du 5 fructidor an III (22 août 1795). L'article 3 de cette Constitution énonce que «la France est divisée en départements». L'article 6 pose le principe selon lequel «les colonies françaises sont parties intégrantes de la République, et sont soumises à la même loi constitutionnelle ». Et l'article 7 tire comme conséquence que les colonies françaises «sont divisées en départements».

La Constitution du 22 frimaire an VIII (13 décembre 1799) remet en question le régime précédent. Elle dispose dans son article 91 que «le régime des colonies est déterminé par des lois spéciales ». C'est dans cette logique que son article premier affirme que «La République française est une et indivisible. Son territoire européen est distribué en départements et

\footnotetext{
${ }^{9}$ Miclo (François), Le régime législatif des départements d'outre-mer et l'unité de la République, Paris, Economica, 1982, p. 26.
} 
arrondissements communaux». La précision «territoire européen » exclut les colonies ultramarines.

C'est au début de la Seconde République que l'on assiste à une nouvelle tentative d'assimilation législative réservée cette fois aux «quatre vieilles » à savoir la Guadeloupe, la Guyane française, la Martinique et la Réunion. Elle émane de Victor Schoelcher qui propose un amendement au cours des débats constitutionnels selon lequel «La présente Constitution est applicable à la Martinique, à la Guadeloupe, à La Réunion et à la Guyane. Ces colonies jouiront du droit commun de la France, sauf les exceptions propres à elles qui seront déterminées par la loi». Le droit commun en matière de division territoriale de la France à l'époque comportait les départements, les arrondissements, les cantons et les communes. Le texte voté fut toutefois très différent de ce qui était souhaité par Schoelcher puisque la Constitution du 4 novembre 1848 disposait dans son article 109 que "le territoire de l'Algérie et des colonies est déclaré territoire français, et sera régi par des lois particulières, jusqu'à ce que une loi spéciale les place sous le régime de la présente Constitution ». La loi spéciale n'a jamais vu le jour.

Dans les «quatre vieilles », un fort courant assimilationniste n'a cessé dès lors de se manifester faisant de l'érection en département le symbole de cette assimilation. Les Conseils généraux de la Martinique et de la Guadeloupe demandent en 1874, 1881 et 1882 la transformation de leur colonie en département. Henri Lémery, sénateur de la Martinique, dépose le 25 janvier 1923 une proposition de loi tendant à transformer en départements français la Martinique, la Guadeloupe, la Guyane et La Réunion ${ }^{10}$. En revendiquant l'effet, à savoir l'érection en département, les auteurs entendent accéder à la cause, à savoir l'assimilation législative, c'est à dire le droit à l'application de plein droit des textes métropolitains.

Les auteurs de la proposition de loi présentée aux députés de l'Assemblée nationale constituante le 26 février 1946 visaient avant tout l'identité législative avec la métropole, l'érection en départements en était la traduction sur le plan administratif. Ils ont obtenu avec la loi votée le 15 mars et promulguée le 19 un classement dans la catégorie des départements, mais sans le droit à l'application automatique des lois et décrets édictés pour la métropole, ce qui constituait pourtant l'essentiel de la revendication dont

${ }^{10}$ Ibidem, p. 53. 
ils étaient les porte-parole. L'application à ces nouveaux départements des lois applicables à la métropole restait subordonnée à des décrets pour les anciennes et à une mention expresse dans la loi pour les nouvelles. Le résultat était le classement dans une catégorie de collectivité de droit commun sans bénéfice de l'ensemble du régime d'identité législative. En disposant que "le régime législatif des départements d'outre-mer est le même que celui des départements métropolitains, sauf exception déterminée par la loi», l'article 73 de la Constitution du 27 octobre 1946 a donné satisfaction aux auteurs de la proposition de loi du 26 février 1946 et mis fin à une incohérence. L'expression doctrinale d'identité législative découle de l'énoncé utilisé dans l'article 73 à savoir «est le même que celui des départements métropolitains ».

Dès lors s'est construit le concept de statut départemental dont le contenu est très précisément celui de la conjugaison d'un régime législatif (celui de l'identité législative avec la possibilité d'exceptions déterminées par la loi) et du classement dans une catégorie de collectivité (les départements). Dans cette conjugaison, le régime législatif est l'élément fondamental. En effet, contrairement à ce que la chronologie des affirmations pourrait faire croire, ce n'est pas parce que les « quatre vielles » sont devenues des départements qu'elles bénéficient de l'identité législative. C'est parce qu'elles bénéficient de l'identité législative qu'elles entrent dans l'organisation administrative de droit commun. Ce n'est pas parce que les « quatre vieilles » ont été érigées en départements que l'application des lois sociales, objet principal de la revendication en 1946, est devenue un droit constitutionnel mais en raison $\mathrm{du}$ régime d'identité législative. L'attachement au statut départemental est donc avant tout l'attachement à un régime législatif. Le classement dans la catégorie des collectivités départementales n'est qu'un effet et non la cause. Les décisions du Conseil constitutionnel relatives à la tentative de mise en place d'une Assemblée unique en $1982^{11}$ et d'une rationalisation de la répartition des compétences entre le département et la région en $1984^{12}$ révèlent que c'est en raison de son interprétation restrictive du champ de modification autorisé par le régime législatif d'assimilation avec possibilité d'adaptation que le Conseil s'est prononcé sur les impossibilités en matière d'organisation administrative

\footnotetext{
${ }^{11}$ Décision n 82-147 du 2 décembre 1982, J.O. 4 décembre 1982, pp. 3666 et 3667.

${ }^{12}$ Décision n ${ }^{\circ} 84-174$ DC du 25 juillet 1984 précitée.
} 
et de répartition des compétences. Ceci démontre que c'est bien le régime législatif l'élément déterminant dans le statut des collectivités situées outre mer. Le régime de l'article 73 de la Constitution de 1958, ancienne rédaction, entendait mettre l'accent sur le champ de l'adaptation ce qui pouvait ouvrir une souplesse dans l'organisation administrative de droit commun et la répartition des compétences ${ }^{13}$. Le Conseil constitutionnel en a jugé autrement préférant faire prévaloir la similitude à un degré plus grand que pour les collectivités situées dans le territoire européen de la France ${ }^{14}$.

$\mathrm{Au}$ fil du temps et du développement de la rhétorique politique, la référence au département s'est chargée d'un contenu plus symbolique et sociologique que juridique ${ }^{15}$. Le mot département en est venu à désigner le territoire lui-même, se substituant dans le langage courant au nom géographique du territoire. Ainsi quelqu'un qui s'absente du territoire martiniquais ne quitte pas la Martinique mais «quitte le département». Il devient ainsi «hors du département». Bien plus, dans nombre de conversations ou de discours, il n'est pas question de Martiniquais, de Guadeloupéens, de Guyanais ou de Réunionnais mais de «domiens». Ce terme n'est pas utilisé pour désigner seulement une origine géographique ou une situation juridique particulière au sein de la République. Il va jusqu'à servir parfois à évoquer une identité...

Une telle identification du territoire martiniquais, guadeloupéen, guyanais et réunionnais au vocable département, ainsi que l'amalgame historique entre département et droit aux avantages sociaux, rendaient extrêmement difficile aux partisans de la réforme de faire comprendre qu'en demeurant régi par le régime de l'identité législative, le droit à l'application de plein droit des lois et règlements était maintenu, et que la substance principale du statut dit « départemental » était préservée.

L'amalgame entre département et avantages acquis se retrouve notamment dans la position défendue par les parlementaires de La Réunion.

13 Le Général De Gaulle, par l'intermédiaire d'André Malraux en déplacement à la Martinique, avait promis d'inscrire des «franchises» dans la constitution en faveur des Départements d'outre-mer.

${ }^{14}$ Voir Douence (Jean-Claude), «Le statut constitutionnel des collectivités d'outre-mer », RFDA, mai-juin 1992, p. 470.

${ }^{15}$ Sur le plan juridique, la collectivité départementale est un type de collectivité territoriale de la République parmi d'autres existantes ou possibles. 
Au cours des débats de l'Assemblée nationale du vendredi 7 novembre 2003, on note l'intervention suivante de $\mathrm{M}$. About : "L'assimilation législative garantit les droits acquis dans le domaine social. Au demeurant, $92 \%$ des Martiniquais souhaitaient il n'y a pas si longtemps, le maintien du département, gage des droits acquis ». D'un côté, le député de La Réunion admet que c'est «l'assimilation législative » qui garantit les droits sociaux, de l'autre, il lie ces droits à l'existence du département comme si «l'assimilation législative » était liée à l'existence de la collectivité départementale. C'est là faire fi de la portée de la révision constitutionnelle. Celle-ci assouplit le régime «d'assimilation » en prenant en compte «les caractéristiques et contraintes ». Elle donne un effet utile à cette prise en compte en rendant possible de demeurer dans «l'assimilation législative» tout en ayant une organisation administrative simplifiée plus en adéquation avec les contraintes de l'exiguïté territoriale. Cette vision consistant à établir un lien indissoluble entre département et «assimilation législative» explique largement l'amendement adopté à la demande du sénateur de La Réunion M. Jean-Paul Virapoullé qui, non seulement enracine constitutionnellement La Réunion dans les collectivités départementale et régionale, mais encore place ces collectivités territoriales en dehors des habilitations possibles à exercer certains pouvoirs. Cette volonté de s'ancrer dans le droit commun ignore délibérément le fait que le droit commun de la République est l'existence de régions pluri-départementales et que l'existence de régions monodépartementales constitue une différence évidente par rapport au droit commun. Pour retrouver le droit commun, dans l'optique d'une assimilation pure et parfaite, il aurait fallu opter pour la bidépartementalisation, ce que les élus réunionnais ont finalement refusé...

Le fait que La Réunion se soit placée en dehors du processus de consultation et que la consultation n'ait pas pu avoir lieu en Guyane a contribué à accroître la méfiance de nombre d'électeurs à qui on a pu faire croire qu'il y avait un mauvais coup qui se mijotait en coulisse contre la Martinique et la Guadeloupe.

La difficulté pour les électeurs d'y voir clair avant de se prononcer est venue non seulement des peurs agitées et du poids de l'histoire mais aussi des interrogations engendrées par le texte de la révision constitutionnelle ${ }^{16}$.

\footnotetext{
${ }^{16}$ Sur la révision constitutionnelle du 28 mars 2003, voir notamment le dossier publié par la RFDA, juillet-août 2003, pp. 661-740 et Faberon (J-Y) (dir.), L'outre-mer français : la nouvelle donne institutionnelle, Les études de la documentation française, 2004, n 5193-5194.
} 


\section{mars 2003 \\ C - Les interrogations résultant du texte de la révision du 28}

Les controverses autour de la consultation du 7 décembre 2003 ont fait surgir un certain nombre de questions et notamment celles-ci :

Que faut-il entendre par statut ? A partir de quand peut-on considérer qu'il y a changement de statut ou simplement réforme du statut, au sens de modification de certains de ses éléments ${ }^{17}$ ? La modification de l'un des éléments du statut, à savoir l'organisation administrative, constitue-t-elle un changement de statut? A partir de quel degré de modification du régime législatif peut-on considérer qu'il y a changement et non pas seulement réforme du statut? Que faut-il entendre par évolution institutionnelle ? Se distingue-t-elle du changement de statut ? Etait-il possible de faire connaître aux électeurs le contenu du statut envisagé ?

L'analyse du texte constitutionnel si elle s'avère parfois éclairante ne l'est pas toujours.

\section{La notion de statut}

L'exposé des motifs de l'avant-projet de loi constitutionnelle expliquait que "l'article 72-3, créé par l'article 7 du projet de loi...offre un choix entre deux options statutaires : le statut de département et de région d'outre-mer, régi par le principe d'assimilation législative; le statut de collectivité régie par le principe de spécialité législative. Le passage de l'un à l'autre de ces statuts est expressément pris en compte et subordonné au consentement des électeurs de la collectivité concernée».

Il résulte de cette rédaction que l'intention du Gouvernement était de distinguer clairement deux statuts à savoir d'une part, le statut de collectivités régies par l'article 73, autrement dit par le régime de l'identité législative avec adaptation, et d'autre part, celui de collectivités régies par l'article 74, autrement dit par le régime de la spécialité législative de principe. Le passage de l'un à l'autre constituait clairement un changement de statut. A contrario, on pouvait penser, à ce stade, que le fait de demeurer dans le même régime ne constituait pas un changement de statut.

${ }^{17}$ Comme on révise la constitution sans en changer. 
Sur la base de ces principes, l'article 72-3 de l'avant-projet de loi constitutionnelle était initialement rédigé de la façon suivante :

«Le statut de la Guadeloupe, de la Guyane, de la Martinique, de Mayotte, de la Polynésie française, de La Réunion, de Saint-Pierre-etMiquelon et des îles Wallis et Futuna est régi soit par l'article 73, soit par l'article 74.

Le passage de tout ou partie de ces collectivités de l'un à l'autre des régimes prévus par les articles 73 et 74 est décidé par une loi organique, qui ne peut intervenir sans que le consentement des électeurs de la collectivité intéressée ait été préalablement recueilli.

La loi détermine le régime législatif et l'organisation particulière des Terres australes et antarctiques françaises. »

Dans cette rédaction on constate que le statut et le régime qui le détermine sont attribués à des territoires géographiques. Par ailleurs, l'importance de l'enjeu du changement de statut est soulignée, d'une part, par la nécessité de recueillir le consentement des intéressés et d'autre part, par l'utilisation de la forme juridique de la loi organique au lieu de la loi ordinaire. Cette rédaction comportait une imprécision quant à la répartition effective de chaque entité géographique nommée entre l'article 73 et l'article 74. Il renvoyait implicitement à la répartition existante. S'il était facile de classer les DOM dans l'article 73 et les TOM dans l'article 74, il n'était pas évident de savoir où se situeraient les collectivités sui generis telles que Mayotte et Saint-Pierre-et-Miquelon.

A ce stade, en s'en tenant à la dualité statutaire établie par l'avantprojet, on pouvait considérer qu'il y avait changement de statut lorsqu'une collectivité quittait l'un des régimes pour entrer dans l'autre. Le fait de changer de collectivité en demeurant dans le régime de l'article 73 constituait-il aussi un changement de statut ? On pouvait penser que la clarté due à l'électorat pouvait conduire à ne pas désigner de la même façon deux processus différents. C'est ce qui explique qu'il a été distingué réforme institutionnelle et changement de statut. L'argument selon lequel il est souhaitable d'éviter l'amalgame en utilisant les mêmes termes pour désigner des réalités différentes a été mis en avant, comme nous le verrons plus loin, par le Sénat à propos de la dénomination de «collectivité à statut particulier $\gg$. Il avait ici aussi sa pertinence. 
L'exposé des motifs du projet de loi reprenait l'essentiel de celui de l'avant-projet, en apportant quelques précisions. Il disait : "le nouvel article 72-3...définit deux catégories statutaires: le statut de département et de région d'outre-mer, régi par le principe d'assimilation législative; le statut de collectivité régie par le principe de spécialité législative. Les actuels départements d'outre-mer continueront de relever de l'article 73 et les autres collectivités de l'article 74. Le passage de l'un à l'autre de ces régimes sera subordonné à l'intervention d'une loi organique, qui devra recueillir le consentement préalable des électeurs de la collectivité intéressée, convoquée par décision du Président de la République ».

Comme précédemment, la notion de statut est utilisée par l'exposé des motifs mais avec quelques différences. L'expression «options statutaires » est remplacée par «catégories statutaires ». Dès lors, on peut en déduire que la première catégorie statutaire est celle de département et région d'outre-mer régis par le principe d'assimilation législative et la seconde catégorie est celle des collectivités régies par le principe de spécialité législative. D'après ce texte, la catégorie statutaire se caractérisant par le régime législatif, il y a changement de catégorie statutaire quand il y a changement de régime législatif.

La rédaction de l'article 72-3 incluse dans le projet de loi faisait apparaître quelques différences avec l'avant-projet. Elle énonçait :

«La Guadeloupe, la Guyane, la Martinique, La Réunion, Mayotte, Saint-Pierre-et-Miquelon, les îles Wallis et Futuna et la Polynésie française sont régis par l'article 73 pour les départements et les régions d'outre-mer, et par l'article 74 pour les autres collectivités.

Aucun passage de tout ou partie de ces collectivités de l'un à l'autre de ces régimes prévus par les articles 73 et 74 ne peut intervenir sans le consentement des électeurs de la collectivité intéressée, convoqués par le Président de la République sur proposition du Gouvernement, ait été préalablement recueilli. En ce cas, le changement de régime est décidé par une loi organique.

La loi détermine le régime législatif et l'organisation particulière des Terres australes et antarctiques françaises.» 
On constate tout d'abord que le terme de statut disparait du texte. Il n'en demeure pas moins que cet article, à la lumière de l'exposé des motifs, doit être compris comme distinguant deux catégories statutaires, chacune étant caractérisée soit par le régime d'identité législative soit par celui de spécialité législative. Ensuite, à l'alinéa premier il est précisé que sont régies par l'article 73 (et donc par le régime de l'identité législative) les entités géographiques qui ont été érigées en départements et en régions d'outre-mer (Guadeloupe, Guyane, Martinique et Réunion). Les autres «collectivités » (au sens d'entités géographiques énumérées nominativement par l'article) relèvent de l'article 74. Enfin, l'autorité investie du pouvoir d'interroger les électeurs concernés par le changement de catégorie statutaire (passage de l'un à l'autre) est précisée. Il s'agit du Président de la République sur proposition du Gouvernement.

Il résulte du texte de l'article 72-3 du projet de loi que le statut des entités géographiques situées outre-mer se caractérise bien par un type d'organisation administrative (un type de collectivité territoriale) et un régime législatif. Il n'y a donc pas lieu de distinguer, voire d'opposer, statut et régime législatif. Cette analyse est corroborée par la rédaction de l'article 74, alinéas un et deux. Il y est écrit :

"Les collectivités d'outre-mer régies par le présent article ont un statut qui tient compte des intérêts propres de chacune d'elles au sein de la République.

Ce statut est défini par une loi organique, adoptée après avis de l'assemblée délibérante, qui fixe :

- les conditions dans lesquelles les lois et règlements y sont applicables ;

- les compétences de cette collectivité...

- les règles d'organisation et de fonctionnement des institutions de la collectivité...

- les conditions dans lesquelles ses institutions sont consultées...

- $\quad \ldots »$

La loi organique, établissant le statut de ces collectivités, précise donc notamment le régime législatif, les compétences et l'organisation 
administrative de la collectivité concernée. Ceci indique que le statut ne se réduit pas au seul aspect de l'organisation administrative et constitue un tout composite.

Les amendements adoptés au cours des débats parlementaires ont opéré plusieurs modifications du texte du projet de loi. S'agissant de l'article 72-3 et de la question statutaire, une des lectures possibles du projet de loi pouvait conduire à considérer que ne pouvaient relever de l'article 73 que les collectivités départementales et régionales. En conséquence, la collectivité se substituant au département et à la région prévue par le dernier alinéa de l'article 73 se trouverait régie par l'article 74 . Un amendement, introduit au Sénat, a permis de lever toute ambiguité en précisant à l'article 72-3 que cette collectivité territoriale est régie, elle aussi, par l'article $73^{18}$. Quoique la rédaction de l'article 72-3 ait levé le doute sur ce point, on a pu constater au cours de la campagne relative à la consultation du 7 décembre 2003 que certains tenants du NON ont instrumentalisé politiquement le texte de l'alinéa premier de l'article 73 en arguant qu'il ne mentionnait que les départements et les régions d'outre-mer comme étant les collectivités territoriales dans lesquelles les lois et règlements sont applicables de plein droit. En conséquence, selon eux, la collectivité se substituant au département et à la région sortait du cadre de l'application de plein droit. Cette instrumentalisation aurait pu être évitée si un amendement avait ajouté à l'article 73 alinéa premier « et dans les collectivités territoriales créées en application du dernier alinéa de cet article ». Vu la rédaction de l'article 72-3, l'éligibilité de la collectivité de substitution au régime de l'application de plein droit ne souffre aucun doute,

\footnotetext{
${ }^{18}$ La ministre de l'outre-mer avait interprété cet article dans ce sens lors de son audition le 16 octobre 2002 devant la commission des lois du Sénat. Toutefois, la rédaction étant ambiguë, un amendement de clarification a été présenté d'une part par M. René Garrec, le 23 octobre 2002, au nom de la commission des lois et d'autre part par trois sénateurs respectivement de la Guadeloupe de la Guyane et de la Martinique, à savoir MM. Larifla, Othily et Désiré, le 28 octobre 2002. Le sénateur martiniquais Claude Lise et les membres du groupe socialiste dans un amendement du 29 octobre 2002 avaient proposé la suppression de l'article 72-3 car il comporte une ambiguïté au regard de l'article 72 et sa rédaction introduit un élément de rigidité préjudiciable à une bonne appréhension des réalités à prendre en compte. "Il supprime toute possibilité de prévoir une réponse adaptée aux aspirations qui s'expriment dans certains départements d'outre-mer et dont la prise en compte nécessite de véritables dérogations au principe d'assimilation législative (dans des domaines tels que par exemple: la culture, l'aménagement du territoire, l'urbanisme, les transports ...).
} 
on pouvait toutefois éviter que l'on puisse induire les électeurs en erreur en le disant aussi dans l'article 73 alinéa premier.

L'éligibilité à l'article 73 de la collectivité créée par la loi se substituant à un département et une région d'outre-mer étant donc clairement avérée, la question se posait de savoir comment qualifier la collectivité ainsi créée et l'acte de substitution au département et à la région. S'agissait-il d'une collectivité à statut particulier? L'acte de substitution opérait-il un changement de statut?

\section{Les différentes approches de la qualification de «collectivité à statut particulier »}

L'instrumentalisation politique de la qualification de collectivité à statut particulier a été particulièrement nette. Pour certains adversaires de la réforme, devenir une collectivité à statut particulier c'était en quelque sorte le début d'un engrenage fatal conduisant au largage ou à la sortie sournoise de la République.

Les controverses doctrinales sur la qualification juridique de la collectivité de substitution au département et à la région d'outre-mer s'expliquent en grande partie par le fait que la qualification de collectivité à statut particulier a fait l'objet de plusieurs modifications dans le processus d'élaboration du texte final.

L'avant projet de loi, dans la version initiale de l'article 73, ainsi que son exposé des motifs qualifiaient la collectivité territoriale "créée en lieu et place d'un département et d'une région d'outre-mer» de collectivité territoriale «à statut particulier ». Ils rattachaient ainsi la collectivité créée à la catégorie prévue à la dernière phrase de l'alinéa premier de l'article 72 de l'avant-projet de loi qui disposait «La loi peut également créer une collectivité à statut particulier, en lieu et place de celles mentionnées au présent alinéa ». L'article 72-1 pour sa part prévoyait la possibilité pour le législateur de consulter les électeurs inscrits dans le ressort des collectivités intéressées lorsqu'il était envisagé de créer une collectivité territoriale dotée d'un statut particulier (au sens de l'article 72 alinéa premier). L'article 74 relatif aux collectivités d'outre-mer qualifiait aussi leur statut de statut particulier. 
La rédaction du projet de loi a fait disparaître la qualification de statut particulier dans l'article 73 dernier alinéa. La qualification était maintenue pour la rédaction proposée de l'article 72 alinéa premier dernière phrase et dans le premier alinéa de l'article 74 . La volonté de changer sciemment la qualification de la collectivité créée en substitution d'un département et d'une région d'outre-mer, autrement dit de ne plus la considérer comme collectivité à statut particulier, est corroborée par l'exposé des motifs qui l'intitule «collectivité nouvelle» sans indiquer sa nature précise. Madame Girardin, ministre de l'outre-mer, au cours des débats parlementaires indiqua cependant que la collectivité créée par la loi en substitution à un département et une région d'outre-mer devait être considérée comme étant une collectivité à statut particulier au sens de l'article 72 alinéa premier ${ }^{19}$. Les amendements du Sénat ont fait évoluer la qualification.

La commission des lois du Sénat a apporté au texte présenté par le Gouvernement plusieurs amendements relatifs à la qualification de statut particulier :

- elle faisait disparaitre la qualification de collectivité à statut particulier de l'article 72 alinéa premier.

- en conséquence, elle proposait la suppression de l'alinéa trois de l'article 72-1 prévoyant la possibilité de consultation des électeurs intéressés lorsqu'il est envisagé de créer une collectivité territoriale à statut particulier. l'article 74 .

le mot particulier accolé à statut était également supprimé dans

Quels étaient les motifs de ces amendements sénatoriaux ?

Selon le rapporteur de la commission des lois du Sénat, "la rédaction proposée par le présent projet de loi, en autorisant, dans deux phrases distinctes, la création soit d'une catégorie de collectivité territoriale soit d'une collectivité à statut particulier en lieu et place de collectivités existantes semble indiquer qu'il sera possible de créer :

- soit une catégorie de collectivités territoriales formant un nouvel échelon sur l'ensemble du territoire, sur le modèle des régions,

\footnotetext{
${ }^{19}$ Débats parlementaires, Sénat, $2^{\text {ème }}$ séance du mercredi 27 novembre 2002.
} 
- soit une collectivité unique, dotée d'un statut particulier et devant désormais, dans un souci de rationalisation, nécessairement se substituer à des collectivités existantes, sur le modèle de la fusion envisagée entre la collectivité territoriale et les deux départements de Corse.

Interrogé par votre rapporteur, le gouvernement a indiqué qu'il fallait au contraire comprendre la première phrase comme une reprise de la jurisprudence $d u$ Conseil constitutionnel, qui autorise la création de catégories regroupant une ou plusieurs collectivités territoriales, et la seconde comme une faculté supplémentaire offerte au législateur de créer des collectivités à statut particulier en remplacement de collectivités existantes. Mais alors, il ne serait pas nécessaire, pour créer une collectivité sui generis d'en supprimer d'autres $»^{20}$.

L'amendement proposé à l'article 72 alinéa premier visait donc, aux dires de la commission, à dissiper l'ambiguïté. Quant à l'amendement relatif à l'article 74, faisant disparaître le mot particulier accolé à statut, il s'expliquait, selon le rapporteur, par la volonté "d'éviter qu'une même expression ne recouvre des significations différentes dans plusieurs dispositions de la constitution $»^{21}$.

Le texte final issu des débats parlementaires, promulgué le 28 mars 2003, n'utilise l'expression statut particulier ni dans l'article 73, ni dans l'article 74. En revanche, la catégorie des collectivités à statut particulier apparaît dans l'énumération des collectivités territoriales de la République effectuée par l'article 72 alinéa premier, première phrase. Les collectivités créées par la loi le cas échéant en lieu et place d'une ou de plusieurs des collectivités énumérées dans la première phrase ne sont pas qualifiées explicitement de collectivités à statut particulier. Que faut-il en conclure ? Le résultat final ne semble pas d'une clarté totale. On peut toutefois tenter une réponse.

Elle n'est pas identique pour les collectivités non situées outre-mer que pour les collectivités situées outre-mer. Pour les premières, la question

\footnotetext{
${ }^{20}$ Sénat, session ordinaire de 2002-2003, annexe au procès-verbal de la séance du 23 octobre 2002, Rapport fait au nom de la commission des lois constitutionnelles, de législation, du suffrage universel, du règlement et d'administration générale sur le projet de loi constitutionnel relatif à l'organisation décentralisée de la République, par M. René Garrec. ${ }^{21}$ Ibidem.
} 
du régime législatif va de soi, alors que pour les secondes il constitue un élément essentiel de leur différenciation.

Pour les collectivités non situées outre-mer, on peut conclure que :

- la jurisprudence du Conseil constitutionnel selon laquelle le législateur peut créer sur la base de l'article 72 alinéa premier, seconde phrase, une catégorie générale de collectivité territoriale ou une collectivité en exemplaire unique est confirmée,

- une collectivité en exemplaire unique peut être une collectivité se substituant à des collectivités existantes, mais ne l'est pas nécessairement,

- ces collectivités en exemplaire unique sont des collectivités à statut particulier.

Pour les collectivités situées outre-mer, les conclusions sont moins évidentes.

Il est acquis d'abord que les collectivités territoriales de la République de Saint-Pierre-et-Miquelon et Mayotte qui faisaient partie de la série des collectivités à statut particulier, avant la révision du 28 mars 2003, sont désormais rangées dans la catégorie constitutionnelle des collectivités d'outre-mer régies par l'article 74. Elles ne sont plus des collectivités à statut particulier au sens retenu désormais par la Constitution.

On admet ensuite que, compte tenu des modifications rédactionnelles, les collectivités d'outre-mer régies par l'article 74, qui ont chacune un statut qui leur est propre, ne sont pas des collectivités à statut particulier.

En revanche, pour la qualification de la collectivité se substituant à un département et à une région d'outre-mer, les interprétations divergent.

La disparition de la mention de collectivité à statut particulier à l'article 73, dernier alinéa, s'expliquerait par la volonté des sénateurs de distinguer ces collectivités non seulement de celles de l'article 74 mais aussi de celles qui sont créées en suivant la procédure de l'article 72-1 troisième alinéa. On peut supposer que, pour les auteurs de la distinction, il fallait justifier l'utilisation de deux procédures différentes de création. Les collectivités évoquées à l'article 72-1 dernier alinéa sont créées, éventuellement après consultation pour avis des électeurs concernés, à l'initiative du législateur. En revanche pour les collectivités évoquées à l'article 73 dernier alinéa, la procédure de création, 
précisée par l'article 72-4, prévoit une proposition du Gouvernement ou une proposition conjointe des deux assemblées parlementaires et une décision du Président de la République de convoquer les électeurs. Une réponse négative contraint à l'abandon du projet. Une telle différence de procédure de création justifierait donc pour les sénateurs que la collectivité territoriale visée à l'article 73 dernier alinéa ne soit pas désignée de la même façon et ne soit donc pas rangée dans la même catégorie statutaire que celles visées à l'article 72-1 dernier alinéa.

Une volonté de différenciation terminologique s'est donc manifestée afin de permettre de distinguer plus clairement des collectivités issues de processus de création différents débouchant sur des collectivités de nature différente. Cette volonté de différenciation terminologique ne justifiait-elle pas qu'il faille aussi trouver un vocabulaire approprié pour opérer une distinction entre le passage du régime de l'article 73 à celui de l'article 74 (et vice versa) et la substitution d'une collectivité nouvelle à un département et une région d'outre-mer ou la mise en place d'une assemblée délibérative commune à un département et à une région d'outre-mer ? Etait-il logique de parler dans les deux cas de changement de statut, alors qu'il ne s'agissait pas de processus de création identiques aboutissant à des collectivités de même nature ?

\section{La recherche d'une qualification appropriée pour désigner l'acte de substitution}

Le passage du régime de l'article 73 à celui de l'article 74 et vice versa étant unanimement dénommé changement de statut, il fallait trouver un vocabulaire approprié pour faire la différence entre ce passage et l'acte de substitution d'une collectivité nouvelle unique à un département et une région d'outre-mer. Pour distinguer ces deux options, de nature très différente, l'expression évolution institutionnelle a été souvent préférée dans les débats à celle de changement de statut pour désigner la substitution d'une collectivité nouvelle à un département et à une région d'outre-mer. Les documents d'orientation adoptés par les élus locaux ont d'ailleurs fait apparaître explicitement la distinction. Le document d'orientation adopté par le Congrès des élus départementaux et régionaux de la Guadeloupe, qui avait opté pour le maintien sous le régime de l'article 73, s'intitulait «document d'orientation sur l'évolution institutionnelle de la Guadeloupe ». Le document d'orientation adopté par le Congrès de la Martinique, qui avait 
opté lui aussi pour le maintien sous le régime de l'article 73, s'intitulait " document d'orientation sur l'avenir institutionnel de la Martinique ». En revanche le Conseil municipal de Saint-Barthélemy, qui avait opté pour le passage à l'article 74, avait approuvé un «document d'orientation sur l'évolution statutaire de Saint-Barthélemy» et le Conseil municipal de Saint-Martin, qui avait fait la même option, avait approuvé un document «d'orientation relatif à l'évolution statutaire de Saint-Martin». La différence de terminologie n'était pas le fruit du hasard. Il s'agissait de s'inscrire dans la logique qui présidait à l'avant-projet de loi distinguant clairement deux types de statuts, celui des collectivités régies par l'article 73 et, celui des collectivités régies par l'article 74 . Cette distinction avait l'avantage d'être simple et claire pour les électeurs et avait un avantage pédagogique évident. Ce vocabulaire, dont on mesure aisément la portée pédagogique, a toutefois soulevé des difficultés juridiques.

Les auteurs de la distinction entre changement de statut et évolution institutionnelle donnaient à l'expression évolution institutionnelle le sens d'évolution des institutions, en entendant par institutions l'organisation administrative. Il s'agissait d'indiquer que le régime de l'application de plein droit étant maintenu, l'objectif recherché était celui d'une rationalisation de l'organisation administrative en la simplifiant. L'expression évolution institutionnelle pouvait toutefois revêtir un autre sens sur le plan juridique. Ce fut le cas dans l'arrêt Feler du Conseil d'Etat.

En effet, le Conseil d'Etat dans son arrêt du 4 décembre 2003 considéra que la question posée par le Président était suffisamment précise «pour indiquer aux électeurs intéressés la portée exacte de leur vote » et la mention dans les visas du document d'orientation approuvé par le Congrès des élus départementaux et régionaux n'était pas « de nature à altérer le caractère loyal et clair de la consultation dès lors que la question posée porte seulement sur le principe de la création, en application de l'article 73 de la Constitution précité, sans faire référence à ce document ou à tout autre projet définissant les modalités de mise en ouvre de ce changement de statut ».

Des tenants du NON à la question posée par le Président de la République ont voulu voir dans cet arrêt la confirmation qu'ils avaient raison en affirmant que la réforme proposée ne constituait pas une évolution institutionnelle mais bien un changement de statut. Cette lecture triomphante ne tient pas compte du fait que, dans cet arrêt, le Conseil d'Etat qualifie aussi 
bien la mise en place d'une collectivité territoriale se substituant à un département et à une région d'outre-mer, tout comme l'institution d'une assemblée délibérante unique pour ces deux collectivités, "d'évolution institutionnelle ». Le considérant cinq commence, en effet, de la façon suivante : "considérant que s'il n'appartient pas au Conseil d'Etat d'apprécier l'opportunité de la décision du Président de la République d'organiser une consultation afin de recueillir, en application de l'article 73 précité de la Constitution, le consentement des électeurs sur le principe de l'une ou l'autre des deux évolutions institutionnelles envisagées par le dernier alinéa de cet article...». L'acte de substitution est donc qualifié ici d'évolution institutionnelle. Faut-il y voir une contradiction consistant à qualifier la réforme de deux façons opposées?

En réalité le Conseil d'Etat n'oppose pas les deux. Il ne donne donc pas raison aux uns contre les autres.

La logique du Conseil d'Etat est qu'il y a changement de statut lorsque l'on met en place une organisation administrative substantiellement différente de celle du droit commun. Cette logique conduit à qualifier la mise en place d'une assemblée délibérante unique pour les collectivités départementales et régionales également de changement de statut. Cette formule s'écarte en effet des caractéristiques de droit commun des collectivités départementales et régionales et débouche sur l'institution de départements d'outre-mer et de régions d'outre-mer à statuts propres.

La conséquence de cette logique est qu'en pratique il s'avère difficile d'indiquer avec un vocabulaire différencié des évolutions institutionnelles de nature différente (passage de l'article 73 à l'article 74 et vice versa ou maintien dans l'article 73 avec une organisation administrative différente), ce qui nuit à l'exigence de clarté requise pour les consultations populaires.

La première solution serait, lorsqu'il s'agit de mettre en place la collectivité de substitution prévue à l'article 73 alinéa sept, d'utiliser la formulation de «modification de l'organisation institutionnelle », comme ce fut le cas pour la consultation des électeurs de Corse ${ }^{22}$.

La seconde solution sémantique serait, en suivant la terminologie suggérée par l'exposé des motifs du projet de loi, de distinguer changement

${ }^{22}$ Loi n $^{\circ} 2003-486$ du 10 juin 2003, JO 133 du 11 juin 2003, p. 9815. 
de « catégorie statutaire » et changement de statut. Il y aurait changement de catégorie statutaire lorsqu'on passe du régime de l'article 73 à celui de l'article 74 ou inversement.

Selon cette option terminologique, au sein de la catégorie statutaire de l'article 74 il y aurait autant de statuts que de collectivités territoriales concernées puisque chacune bénéficie d'un statut spécifique défini par une loi organique. Au sein de la catégorie statutaire de l'article 73, il y aurait premièrement le statut ordinaire de département et région d'outre-mer, ensuite le statut de département et région d'outre-mer propre à La Réunion, puis le statut de collectivité unique se substituant à un département et une région d'outre-mer, et enfin le statut propre de département et région d'outre-mer avec Assemblée délibérante unique.

\section{L'impossibilité juridique de consulter les électeurs sur le contenu du statut envisagé}

Le slogan «nous pas Ka acheté chat dans sac » largement utilisé par les partisans du NON a eu un impact incontestable sur l'opinion publique, alimentant la méfiance de nombreux électeurs et entraînant dans une certaine mesure le changement de position de certains partisans initiaux du OUI.

Les électeurs guadeloupéens et martiniquais n'ont été consultés officiellement que sur le principe de la substitution au département et à la région d'une collectivité demeurant régie par l'article 73 . Il s'agissait là d'une exigence juridique selon le Conseil d'Etat.

En effet, dans un premier temps, le Gouvernement avait l'intention de proposer au Président de la République de soumettre aux électeurs non seulement le principe de la substitution en Guadeloupe et en Martinique d'une collectivité régie par l'article 73 au département et à la région mais aussi les documents d'orientation sur l'évolution institutionnelle adoptés par les élus locaux après les corrections apportées à ces documents à la demande du Ministre de l'outre-mer afin de conformer le contenu des projets proposés à la Constitution. C'est ainsi que dans un courrier adressé au président de la région Martinique en date du 16 mars 2003, Madame Girardin indiquait :

"Lors de mon dernier déplacement en Martinique, les 15 et 16 janvier dernier, vous m'avez remis un document relatif à la réforme du statut 
de la Martinique qui synthétise les propositions du Congrès des élus départementaux et régionaux sur l'évolution institutionnelle de l'île.

Ainsi que j'en avais alors pris l'engagement, j'ai l'honneur de vous faire connaître, par la présente, mes observations sur ce texte, alors que le Congrès du Parlement vient de procéder à la ratification du projet de loi constitutionnelle relatif à l'organisation décentralisée de la République.

Ce document doit inspirer le texte du futur «document d'orientation » qui, une fois les conditions politiques réunies, serait soumis au suffrage des électeurs de la Martinique, si le chef de l'Etat en décidait ainsi sur la proposition que pourrait lui faire le Gouvernement.

Ce document d'orientation, dans sa rédaction définitive, pourra être élaboré d'un commun accord entre le gouvernement et les élus de la Martinique, dès lors que le document que vous m'avez remis le 15 janvier 2003 aura lui-même été précisé et complété afin de répondre aux observations qui suivent :

Ces observations porteront successivement sur l'opportunité de certaines dispositions institutionnelles, sur les précisions qu'il convient d'apporter aux compétences de la nouvelle collectivité, et enfin sur la constitutionnalité des dispositions du document... »

Il est indiqué clairement dans cette lettre que le «document d'orientation» serait soumis au suffrage des électeurs de la Martinique. Il est évident, également, que la notion même de « document d'orientation » supposait l'existence d'un texte, définissant les grandes lignes de la réforme, qui, après avoir été négocié entre le Gouvernement et les porte-parole du Congrès des élus départementaux et régionaux, et approuvé par les électeurs, devait servir de base à l'élaboration d'une loi qui serait soumise au Parlement. Celui-ci demeurant libre de l'accepter, de la rejeter ou d'en modifier le contenu. S'il ne s'agissait pas de cela, on ne voit pas quel aurait été l'intérêt de ce document dit d'orientation. Orientation en vue de quoi, si ce n'est de l'élaboration d'une loi ? Que s'est-il donc passé, expliquant que les électeurs n'ont pas eu juridiquement le droit de se prononcer sur le « document d'orientation »?

Avant de formuler, par une lettre en date du 29 octobre $2003^{23}$, la proposition au Chef de l'Etat de consulter les électeurs, le Premier ministre

${ }^{23} J O$ n $^{\circ} 252$ du 30 octobre 2003, p. 18535. 
avait consulté le Conseil d'Etat. Celui-ci, dans un avis du 23 octobre 2003, estima que «... les documents qui seront adressés par l'administration aux électeurs ne doivent contenir aucune information que celle relative aux conséquences qui résulteraient nécessairement, en application de la Constitution elle-même, du changement proposé, quelles que puissent être, par ailleurs, les modalités du statut de la nouvelle collectivité qui seront ultérieurement fixées par le législateur... $\gg^{24}$. S'agissant du passage du régime de l'article 73 à celui de l'article 74 , il a estimé que, selon le texte constitutionnel, "Le consentement des électeurs ne devait porter que sur le principe du changement de l'un vers l'autre des régimes prévus par les articles 73 et 74 de la Constitution et non sur les modalités d'un tel changement, qui doivent être décidées par la loi organique $»^{25}$.

Cette interprétation du texte constitutionnel, selon laquelle les électeurs ne devaient être consultés que sur le principe de la substitution et non sur les orientations qui présideraient à l'élaboration du statut de la collectivité, a été réitérée très explicitement dans l'arrêt Feler. Dans cet arrêt, le Conseil d'Etat a considéré que la mention dans les visas du document d'orientation approuvé par le Congrès des élus départementaux et régionaux n'était pas «de nature à altérer le caractère loyal et clair de la consultation dès lors que la question posée porte seulement sur le principe de la création, en application de l'article 73 de la Constitution précité, sans faire référence à ce document ou à tout autre projet définissant les modalités de mise en cuvre de ce changement de statut $»$.

Le Parlement n'est obligé de se conformer au choix des électeurs qu'en cas de refus par eux de la réforme ${ }^{26}$.

M. Michel Verpeaux commente la position du Conseil d'Etat en écrivant :

"Le Conseil d'Etat considère que les électeurs n'ont pas à se prononcer sur un projet précis. Il approuve le fait que le document d'orientation n'ait pas été annexé à la convocation car il aurait semblé lier le

\footnotetext{
${ }^{24}$ Avis du Conseil d'Etat en 2003, Section de l'intérieur - Avis n 369.561 - 23 octobre 2003, Rapport public du Conseil d'Etat pour 2004, pp. 225-228.

${ }^{25}$ Ibidem.

${ }^{26}$ Sur l'arrêt du Conseil d'Etat, on peut se reporter à Thiellay (Jean-Philippe), La consultation des électeurs des départements français des Antilles du 7 décembre 2003, note sous Conseil d'Etat, 4 décembre 2003, Feler, RFDA, mai-juin 2004, pp. 549-552.
} 
Parlement, seul compétent pour adopter le nouveau statut de la collectivité. Les électeurs ne devaient se prononcer que sur le principe du changement statutaire sans en connaître les éléments précis $»^{27}$.

Dans son avis préalable au décret présidentiel et dans l'arrêt Feler le Conseil d'Etat a indiqué qu'il fallait respecter les prérogatives du Parlement à qui il appartiendrait de légiférer en toute liberté sur le contenu du statut de la collectivité nouvelle. Cette position est dans la ligne de celle du Conseil constitutionnel qui, dans sa décision $n^{\circ} 87-226$ DC du 2 juin 1987, relative à la consultation des populations intéressées de Nouvelle-Calédonie et dépendances, avait considéré qu'il ne fallait pas laisser croire aux électeurs "que les éléments du statut sont d'ores et déjà fixés, alors que la détermination de ce statut relève, en vertu de l'article 74 de la Constitution, d'une loi prise après consultation de l'Assemblée territoriale $»^{28}$.

Comment comprendre alors qu'il n'était pas possible de faire, en matière d'information officielle sur le contenu envisagé des réformes, pour les électeurs de la Guadeloupe et de la Martinique mais aussi de Saint-Barthélemy et de Saint-Martin qui n'étaient pas eux non plus censés connaître officiellement le contenu des lois organiques précisant leur statuts dans le cadre de l'article 74, ce qui avait pu être fait pour les électeurs de Corse ? En effet, ceux-ci ont pu, avant leur vote du 6 juillet 2003, dans une annexe à la loi du 10 juin 2003 organisant la consultation pour avis ${ }^{29}$, prendre connaissance des éléments relativement précis de la réforme envisagée ?

Les consultations en Corse et aux Antilles françaises n'ont pas été réalisées sur les mêmes fondements constitutionnels. La consultation a été organisée pour la Corse sur la base de l'article 72-1 de la Constitution alors que les consultations antillaises l'ont été en référence aux articles 72-4 et 73. Dans le premier cas, l'initiative de la consultation relevait du législateur, elle portait sur «la modification de l'organisation institutionnelle de la Corse », et visait à recueillir un simple avis. Dans le second cas, celui des Antilles

\footnotetext{
${ }^{27}$ Verpeaux (Michel), «Les consultations locales outre-mer, suite ...et fin ?», AJDA $\mathrm{n}^{\circ}$ 11/2004, 22 mars 2004, p. 597. Selon l'auteur la question posée n'était pas absolument claire et loyale. Voir aussi du même auteur : libres propos sur des consultations locales récentes ou les dérapages de la démocratie locale directe, AJDA 2003, p. 2249.

${ }^{28}$ Décision $n^{\circ}$ 87-226 DC du 2 juin 1987, Rec p. 34.

${ }^{29}$ Loi $^{\circ} 2003-486$ du 10 juin 2003 organisant une consultation des électeurs de corse sur la modification de l'organisation institutionnelle de la Corse, $J O$ du 11 juin 2003, p. 9815. A noter que cette loi n'a pas été soumise au Conseil constitutionnel.
} 
françaises, les consultations relevaient du Président de la République, portaient sur le principe de la création de collectivités nouvelles, les unes régies par l'article 73 (Guadeloupe et Martinique) les autres par l'article 74 (Saint-Barthélemy et Saint-Martin), et visaient à recueillir le consentement des électeurs.

La dissymétrie, préjudiciable en l'occurrence à l'électorat antillais, vient du texte constitutionnel lui-même qui a tenté, sans y parvenir de façon satisfaisante, de concilier plusieurs objectifs: rester cohérent avec les principes énoncés dans l'article 3 de la Constitution, respecter les compétences du Parlement et rassurer les électeurs d'outre-mer en leur donnant un pouvoir de blocage vis-à-vis des changements statutaires qui ne correspondraient pas aux souhaits de la majorité d'entre eux, ce qui peut s'analyser comme un pouvoir d'autodétermination négative.

Cette impossibilité juridique de soumettre aux électeurs antillais des projets précis aurait pu être en partie surmontée par des indications apportées par le Gouvernement au cours de la campagne comme l'y engage d'ailleurs l'avis précité du Conseil d'Etat: «En revanche, - dit-il - il est loisible au Gouvernement de faire connaître par ailleurs, notamment dans le cadre de la campagne précédant le scrutin, les orientations, qu'il envisage pour la mise en cuvre de la réforme proposée aux électeurs, s'agissant par exemple de l'organisation, des compétences ou du régime législatif de la nouvelle collectivité. Mais ces orientations ne sauraient engager juridiquement le législateur, auquel il appartiendra, le cas échéant, de mettre en ouvre la réforme envisagée » ${ }^{30}$. Si le Gouvernement l'avait fait, cela aurait contribué à clarifier la situation. Il n'a pas souhaité le faire au motif qu'il voulait mettre en évidence qu'il s'agissait d'un projet qui n'émanait pas de lui mais des élus locaux. On peut supposer aussi que les consultations antillaises venant après le NON à la consultation en Corse, où il s'était engagé en faveur du changement, il n'a pas voulu prendre le risque d'un nouvel échec.

Ceci étant, l'argument de la méconnaissance par l'électorat des aspects essentiels des réformes envisagées mérite d'être fortement relativisé. L'information sur le contenu des documents d'orientation avait été très

${ }^{30}$ Comme l'indique Jean-Philippe Thiellay «l'avis établit une distinction nette entre l'analyse juridique et la dimension politique du débat », article cité p. 551. 
largement diffusée, même si on aurait pu souhaiter qu'ils aient été envoyés à chaque électeur.

Par ailleurs, n'y avait-il pas une contradiction dans le fait de dénoncer un manque de connaissance du contenu de la réforme (Chat dans sac) et de la contester sur des points précis ? Comment reprocher à la réforme d'opérer un changement de statut en soutenant en même temps qu'on ne savait pas en quoi consistait cette réforme? Plus encore, comment arguer d'un abandon de l'identité législative avec possibilité d'adaptation et d'un basculement dans l'autonomie, dénoncée comme antichambre de l'indépendance, si on ne faisait pas prévaloir une lecture orientée du contenu de la réforme proposée ?

Dans les différents documents d'orientation adoptés par les Congrès des élus départementaux et régionaux de la Guadeloupe et de la Martinique qui ont tenu compte de la réforme constitutionnelle et des préconisations de la Ministre de l'outre-mer, il s'est agi notamment de faire ressortir la distinction essentielle entre le choix du régime de l'article 73 et celui de l'article 74 . Le doute a pourtant été jeté pendant la campagne sur l'effectivité de la distinction. L'argument avait pour objectif d'alimenter l'idée selon laquelle la mise en place de la collectivité nouvelle creusait l'écart avec le droit commun de la République, autrement dit éloignait substantiellement de l'identité législative. En réalité, l'argument en se focalisant sur la collectivité de substitution occultait le fait que s'il y avait un écart accru en matière de régime législatif en vertu des nouvelles dispositions de l'article 73 , celui-ci ne concernait pas spécifiquement la collectivité nouvelle mais aussi les collectivités départementales et régionales régies par l'article $73^{31}$.

Au-delà de l'instrumentalisation politique, il existe toutefois un débat doctrinal sur la portée exacte des modifications apportées par la révision du 28 mars 2003 à la rédaction de l'article 73.

\footnotetext{
${ }^{31}$ La Réunion mise à part s'agissant du pouvoir d'adopter des règles après habilitation par la loi.
} 


\section{La question de la nature du régime juridique du nouvel article 73 de la Constitution}

La stratégie d'un certain nombre de partisans du rejet de la réforme consistait assez largement à agiter l'épouvantail de l'autonomie comme antichambre de l'indépendance. L'indépendance signifiant la rupture avec la France et la Communauté européenne, avec pour conséquence la régression économique et sociale et la dérive vers le despotisme politique. Il fallait ainsi faire croire que la mise en place de la collectivité nouvelle constituait un pas irréversible vers l'autonomie et donc vers l'indépendance. L'option proposée de demeurer sous le régime de l'article 73 aurait pu faire obstacle à l'argument. L'obstacle a été contourné par l'affirmation selon laquelle le régime de l'article 73 modifié gommait les différences avec celui des collectivités régies par l'article 74 , à tel point qu'il ne serait plus exact de parler d'identité législative pour caractériser le régime de l'article 73 . Pour analyser cette question, il convient de rappeler d'abord le contenu de la demande qui était exprimée localement par la majorité des élus avant la révision puis d'apprécier les possibilités offertes par le texte de la Constitution révisée.

\section{A - La demande d'un renforcement substantiel du pouvoir local en vue du développement}

Depuis l'échec de la tentative de rationalisation de l'organisation administrative des départements et régions d'outre-mer, en 1982, puis de la répartition des compétences entre les collectivités départementale et régionale en 1984, le débat sur les voies et moyens susceptibles de permettre de résoudre la question était devenu récurrent. A ceci s'ajoutait une conjoncture de crise économique et sociale. Celle-ci se caractérisait premièrement par les difficultés rencontrées par les productions d'exportation traditionnelles (notamment la banane) ainsi que le secteur touristique, deuxièmement par un taux de chômage très important et troisièmement par une quête d'affirmation identitaire.

Dans ce contexte, le Premier ministre M. Lionel Jospin confia à deux parlementaires ultramarins le soin de faire des propositions. Dans une lettre 
adressée au sénateur Claude Lise en date du 10 décembre 1998, le Premier ministre indiquait que le Gouvernement souhaitait à l'intérieur du cadre de l'article 73 et de l'article 299 paragraphe 2 du traité instituant la Communauté européenne, dans sa rédaction issue du Traité d'Amsterdam, "que soient recherchées les évolutions permettant une efficacité accrue de l'action publique ». Selon cette lettre, inscrire les départements d'outre-mer dans une logique de développement durable constituait une priorité pour le Gouvernement. La mission confiée était de dresser un bilan des avantages et inconvénients du cadre législatif et réglementaire actuel et de faire des propositions premièrement sur la possibilité de nouveaux transferts de l'Etat vers les différents niveaux de collectivités territoriales, deuxièmement sur l'opportunité de modifier la répartition des compétences entre les régions et les départements et troisièmement sur les moyens d'obtenir une meilleure insertion des départements d'outre-mer dans leur environnement régional. Ces propositions devaient être élaborées sur la base d'une large consultation au niveau local. Elles avaient vocation à inspirer la rédaction d'un projet de loi d'orientation.

Le rapport issu du travail des deux parlementaires a été remis au Premier ministre en juin 1999 et s'intitulait «Les départements d'outre-mer aujourd'hui : La voie de la responsabilité $»^{32}$. Les propositions du rapport se résumaient autour de sept points, à savoir : la création d'un Congrès : la voie d'une évolution statutaire transparente et démocratique ; l'accroissement des responsabilités locales: condition du développement; la clarification des compétences : condition d'une meilleure lisibilité des politiques publiques ; le rétablissement financier : condition des libertés locales ; l'adaptation du droit : condition de l'efficacité de l'action publique; la déconcentration de l'Etat: condition d'un vrai partenariat ; l'article 299\$2 : condition d'une intégration économique rénovée.

Le Congrès proposé était la réunion des assemblées départementales et régionales. Il était destiné d'une part à gérer les compétences partagées dans les domaines des transports, du logement, et de l'aménagement du territoire, et d'autre part à initier un éventuel processus de changement statutaire devant être soumis à la population concernée. La proposition allait au-delà de la lettre de mission demandant de s'inscrire dans le cadre de l'article 73.

${ }^{32}$ Lise (Claude) et Tamaya (Michel), Les Départements d'outre-mer aujourd'hui : la voie de la responsabilité, Paris, la Documentation française, 1999. 
Dans un texte daté du 1 décembre 1999, intitulé «Déclaration de Basse-Terre », les présidents des régions de Guadeloupe, Guyane et Martinique ont fait rebondir la question à la fois du développement et de l'évolution statutaire de leurs pays. Après avoir brossé le tableau de la "dégradation continuelle depuis de nombreuses années de la situation de leurs régions », ils ont affirmé leur volonté de "bâtir un projet de développement économique, social et culturel impliquant la prise en compte des identités propres à chaque région...». Après avoir décliné les éléments essentiels de ce projet de développement, les signataires ont demandé au Président de la République et au Gouvernement «une modification législative, voire constitutionnelle, visant à créer un statut nouveau de Région d'Outre-mer doté d'un régime fiscal et social spécial» pour leurs pays respectifs et ceci «dans le cadre de la République française d'une part, et de l'Union européenne d'autre part (article 299-2 du Traité d'Amsterdam)».

Ce texte appelle, entre autres, les observations suivantes : il établit, lui aussi comme le rapport Lise Tamaya, un lien entre développement et réforme statutaire; il préconise la mise en place d'une Région d'Outre-mer doté d'un régime fiscal et social spécial sans autres précisions sur la nature de la nouvelle collectivité (la référence aux résultats obtenus par les autres régions ultrapériphériques peut laisser supposer que le statut nouveau pourrait s'inspirer du leur mais ce n'est pas dit explicitement); il ne précise pas la procédure d'élaboration souhaitable de ce statut ni la place qui devra être faite à la consultation des électeurs concernés ; et il s'inscrit dans la perspective d'une révision constitutionnelle éventuellement nécessaire.

En contrepoint à la déclaration de Basse-Terre, les trois présidents des Conseils généraux de Guadeloupe, de Guyane et de Martinique ont signé, le 8 février 2000, un communiqué commun préconisant une série de mesures économiques et sociales urgentes et réaffirmant "leur attachement au Congrès...lieu de débat légal, transparent et démocratique, ainsi qu'à la consultation des populations sur tous projets d'évolution statutaire».

Le 11 mars 2000, le Président de la République, dans son discours de Madiana, a admis les limites du statut départemental et l'éventualité d'une révision constitutionnelle afin de répondre aux nécessités d'évolution sous réserve de l'accord des populations concernées. 
Parallèlement à l'élaboration de la loi d'orientation pour l'outre-mer, la région Martinique avait mis en chantier l'élaboration du «Projet Martinique ». Un forum-débat de synthèse de sept ateliers composés d'élus de différentes tendances, de socioprofessionnels et de participants divers, fut organisé à Madiana le 2 décembre 2000. Le projet comportait un volet économie et développement durable et solidaire, un volet social, un volet culture et sport, un volet environnement-aménagement, un volet éducationformation, un volet coopération régionale et internationale et bien entendu un volet institutions. Ce dernier volet proposait un dispositif institutionnel, des pouvoirs accrus et de nouveaux domaines de compétences. La nouvelle collectivité devait comporter une assemblée dont le mode d'élection serait choisi entre plusieurs possibilités de proportionnelle, un conseil exécutif distinct de l'Assemblée et responsable devant elle, et une Assemblée consultative. Parmi les pouvoirs escomptés, il y avait ceux d'adapter les textes règlementaires, de déroger à certaines dispositions législatives par des délibérations de valeur réglementaire non soumises à une validation du parlement. La justice devenait un domaine partagé entre l'Etat et la collectivité nouvelle. L'hypothèse que l'Assemblée puisse être dotée d'un véritable pouvoir législatif a été évoquée par l'atelier institutions. Il était établi pour les initiateurs du projet qu'il fallait aller au-delà de ce que permettait en l'état l'article 73 et que l'évolution envisagée devait passer par une révision constitutionnelle et obtenir la ratification du peuple martiniquais.

La loi d'orientation pour l'outre-mer promulguée le 13 décembre $2000^{33}$ s'était fixée comme objectif essentiel de «promouvoir le développement durable» des départements d'outre-mer au moyen d'une série de mesures dans les domaines économique, social et culturel. Elle entendait favoriser le développement de leurs relations internationales et reconnaissait "à la Guadeloupe, à la Guyane, à la Martinique et à La Réunion la possibilité de disposer à l'avenir d'une organisation institutionnelle qui leur soit propre ». La proposition de mise en place d'un Congrès des élus départementaux et régionaux figurant dans le rapport Lise Tamaya, néanmoins revue et corrigée afin de la rendre compatible avec les exigences constitutionnelles, a été retenue par la loi d'orientation. Le Congrès s'est vu reconnaître la compétence de délibérer "de toute proposition d'évolution institutionnelle, de toute proposition relative à de

\footnotetext{
${ }^{33}$ Loi n $^{\circ}$ 2000-1207 du 13 décembre 2000, J.O. du 14 décembre 2000, p. 19760.
} 
nouveaux transferts de compétence de l'Etat vers le département et la région concernés, ainsi que de toute modification de la répartition des compétences entre ces collectivités locales ${ }^{34}$. Le champ ainsi ouvert supposait l'acceptation implicite d'aller au-delà de ce que permettrait l'article 73 dans sa rédaction du moment et donc d'opérer une révision de la Constitution. Quant à la consultation de la population de la collectivité concernée, la loi d'orientation prévoit que le Gouvernement peut déposer un projet de loi organisant une consultation pour recueillir son avis. Il n'est pas obligé de le faire et l'avis ne le lie pas.

En Martinique, conformément à la procédure prévue par la loi d'orientation pour l'outre-mer, le Congrès des élus départementaux et régionaux s'est réuni tour à tour sous la présidence du président du Conseil général et du président du Conseil régional. Les réunions ont été préparées par la mise en place d'une commission dite ad hoc reflétant les diverses tendances politiques représentées au sein des deux assemblées. Le projet Martinique a servi de base de discussion au départ.

Il est ressorti des travaux du Congrès un certain nombre de propositions et de prises de positions (sous forme de motions) adoptées lors de ses séances des 20,21, 23 février et 4 mars 2002, ceci à des majorités plus ou moins importantes.

Au nombre des propositions, il convient de retenir :

- L'érection de la Martinique en une nouvelle collectivité territoriale dans le cadre de la République française et de l'Union européenne (pour 64, contre 17, abstention 1 et ne prenant pas part au vote (ci-après NPPPV 0 ).

- La mise en place au sein de cette collectivité d'une Assemblée unique dotée de pouvoirs accrus (pour 62, contre 0, abstention 0 et NPPPV 19).

- L'Assemblée composée de 75 membres dispose d'un président élu en son sein au scrutin majoritaire. Celui-ci exerce la fonction exécutive assisté d'une commission élue à la proportionnelle. Sa responsabilité peut être mise en cause par une motion de défiance dont les auteurs doivent proposer un nouvel exécutif. Quatre conseils consultatifs sont placés auprès de la nouvelle collectivité : un conseil économique et social, un conseil de la

\footnotetext{
${ }^{34}$ Article 62 de la loi d'orientation pour l'outre-mer.
} 
culture, de l'éducation et de l'environnement, un conseil de la famille, de la jeunesse et de la démocratie participative, et un conseil des communes assurant la représentation du territoire (pour 56, contre 0 , abstentions 2 et NPPPV 7).

- L'attribution à la nouvelle collectivité de compétences supplémentaires (pour 63, contre 0, abstention 0, NPPPV 13).

- La dotation des moyens permettant à la collectivité nouvelle d'exercer ses compétences supplémentaires (unanimité des 76 élus présents et représentés).

- Le transfert de l'Etat à la nouvelle collectivité des compétences supplémentaires suivantes : définir et gérer la politique du logement et de l'habitat ; définir et mettre en œuvre la politique de planification spatiale et de l'urbanisme; définir et adapter les politiques d'aménagement aux réalités locales ; élaborer sa politique énergétique, mettre en œuvre un programme de diversification et de valorisation des ressources énergétiques renouvelables, édicter les règles et les méthodes applicables aux constructions en prenant en compte les risques naturels majeurs et technologiques inhérents à la Martinique, définir le cadre de l'élaboration des plans de prévention des risques naturels majeurs et technologiques, élaborer le classement et le déclassement en zone de réserve naturelle et préserver les diversités biologiques et les biotopes; veiller à la mise en place d'une politique de l'environnement et de l'écologie, intégrer dans le patrimoine de la collectivité les cours d'eau, les ressources du sous-sol ainsi que les terrains acquis par le conservatoire du littoral ; transférer au patrimoine de la collectivité les sites, les monuments historiques et archéologiques et le sous-sol ; définir sa politique culturelle, artistique et sportive en partenariat avec les associations et les organismes agissant dans ces domaines; participer à la définition de la politique foncière publique et à la gestion des 50 pas géométriques, le domaine public de l'Etat étant intégré au patrimoine de la Collectivité, l'intégration au patrimoine de la Collectivité des îlets dont les titres de propriété ne sont pas encore validés; assurer la gestion du sous-sol à travers la mise en œuvre du code minier ; participer à l'élaboration de la politique des transports aériens, maritimes et terrestres, la mise en place d'une seule autorité organisatrice du transport à vocation multimodale et intermodale, le droit à être consulté pour la délivrance des droits de trafic maritime et aérien (unanimité des 76 élus présents et représentés). 
- L'attribution à la nouvelle collectivité territoriale d'un pouvoir d'adaptation ou de modification des décrets d'application des lois votées au Parlement ainsi qu'un pouvoir de proposition de décrets d'application des lois (pour 53, contre 1, abstentions 19).

- L'attribution à la nouvelle collectivité territoriale d'un pouvoir propre d'adoption de décrets dans le cadre de ses compétences (pour 53, contre 0 , abstentions 19$)$.

- L'attribution à la nouvelle collectivité d'un pouvoir législatif encadré obtenu par habilitation et permettant à l'Assemblée unique de légiférer dans certains domaines, dans les conditions fixées par le Parlement (pour 52, contre 19, abstentions 11).

- L'élection de l'Assemblée unique dans le cadre d'une circonscription unique et sur la base d'un scrutin proportionnel assorti d'un seuil de $5 \%$ des suffrages exprimés (pour 72 , contre 0 , abstention 0 ).

A ces propositions ont été ajoutées trois motions tendant à :

- La reconnaissance de l'existence d'un peuple martiniquais (pour 45, contre 0 , abstentions 3, NPPPV 25).

- La reconnaissance de l'existence d'une nation martiniquaise (pour 23, contre 11, abstentions 2, NPPPV 38).

- Garantir le maintien de la nouvelle collectivité dans l'Union européenne, assorti de la possibilité de prendre en sa faveur des mesures de discrimination positive (pour 82, contre 0 , abstention 0 , NPPPV 1 ).

Les propositions du Congrès des élus départementaux et régionaux de la Martinique ont été transmises, conformément à la loi d'orientation pour l'outre-mer ${ }^{35}$, au Conseil général, au Conseil régional et au Premier ministre. Après saisine des deux conseils consultatifs, les propositions du Congrès ont fait l'objet d'une approbation par les conseils général et régional. Enfin, chaque collectivité a transmis sa délibération au Premier ministre.

Ces propositions sortaient bien évidemment du cadre des possibilités offertes par l'article 73 à savoir l'identité législative avec adaptation et même d'une façon plus large de ce qu'autorisait l'article 72, autrement dit la

\footnotetext{
${ }^{35}$ Article 62.
} 
possibilité de création d'une collectivité nouvelle sui generis demeurant dans le cadre de la libre administration. L'existence d'un pouvoir législatif même encadré allait beaucoup plus loin que ce qu'autorisait la Constitution.

Le document d'orientation acté par les élus locaux le 10 avril 2003 a mis en concordance les propositions issues du Congrès avec les possibilités offertes par le texte révisé de l'article 73.

L'analyse du texte constitutionnel fait ressortir que les assouplissements apportés à l'article 73 sont assortis de limites d'une importance telle qu'il n'y a pas lieu de considérer qu'ils brouillent totalement les frontières entre la catégorie statutaire de l'article 73 et celles de l'article 74.

\section{B - Des assouplissements assortis de limites essentielles}

Les assouplissements apportés par la révision constitutionnelle du 28 mars 2003 tiennent compte de certaines demandes exprimées par les Congrès de Guadeloupe et Martinique. L'objectif recherché semble avoir été de rendre attractif la catégorie statutaire de l'article 73 pour les collectivités déjà régies par cet article. Les assouplissements consistent à étendre le champ de l'adaptation des textes nationaux, à permettre au législateur d'habiliter les élus locaux à décider des adaptations des textes nationaux dans les matières où s'exercent leurs compétences et à adopter « des règles applicables sur leur territoire dans un nombre limité de matières pouvant relever du domaine de la loi ». nationales

- L'extension du champ de l'adaptation décidée par les autorités

L'application de plein droit est modulée par les possibilités d'adaptations ouvertes pour le législateur et le pouvoir réglementaire central. $\mathrm{Vu}$ le risque d'interprétation restrictive qui a prévalu dans la jurisprudence du Conseil constitutionnel, le texte révisé ne se réfère plus comme dans l'article 73 ancien aux «mesures d'adaptation nécessitées par leur situation particulière». Il utilise une formule plus large à savoir "tenant à leurs caractéristiques et contraintes ». Cette rédaction s'inspire de l'article 299 paragraphe 2 du Traité instituant la Communauté européenne qui stipule que "les dispositions du présent traité sont applicables aux départements d'outre-mer... », qui admet la possibilité de «mesures spécifiques» et des 
«conditions d'application» décidées par le Conseil en raison de «la situation économique et sociale structurelle » des régions ultrapériphériques, aggravée par une série de facteurs permanents qui combinés entre eux «nuisent gravement à leur développement», et qui prévoit que «le Conseil arrête les mesures visées au deuxième alinéa en tenant compte des caractéristiques et contraintes particulières » de ces régions. nationaux

- L'attribution d'un pouvoir décentralisé d'adaptation des textes

Une nouveauté consiste à donner aux collectivités territoriales concernées la possibilité, par une habilitation législative, de faire elles-mêmes les adaptations dans leurs champs de compétence. L'alinéa 4 précise que «les habilitations sont décidées à la demande de la collectivité concernée». Le texte de la Constitution révisée renvoie à une loi organique pour plus de précisions sur les modalités de mise en œuvre de ce nouveau pouvoir.

- L'attribution d'un pouvoir de fixation de règles applicables sur le territoire des collectivités concernées dans des matières pouvant relever du domaine de la loi

Le texte de la révision prévoit également la possibilité d'une habilitation législative au profit de la collectivité demandeuse lui permettant de fixer elle-même les règles applicables y compris dans certaines matières relevant du domaine de la loi. On va ainsi au-delà de l'adaptation. Il s'agit d'une dérogation à la Constitution donnée par la Constitution elle-même. Les habilitations prévues sont décidées, comme précédemment, dans les conditions et sous les réserves prévues par une loi organique.

Les assouplissements introduits par la révision dans le régime législatif des départements et régions d'outre-mer connaissent un certain nombre de limites ${ }^{36}$ qui ont pour effet de relativiser leur portée et de maintenir une différence essentielle entre le régime de l'article 73 et celui de l'article 74.

- L'adaptation demeure limitée par nature et n'est pas obligatoire

En permettant au législateur et au pouvoir réglementaire de ne procéder qu'à des adaptations, le texte de la Constitution révisée offre moins

\footnotetext{
${ }^{36}$ Pour une analyse de ces limites voir également Roux (André), Le pouvoir normatif, in L'outre-mer français. La nouvelle donne institutionnelle, opus cit., pp.134-136.
} 
de possibilités que l'article 299 paragraphe 2 du traité instituant la Communauté européenne (traité CE) qui donne pouvoir au Conseil d'arrêter «des mesures spécifiques visant, en particulier, à fixer les conditions de l'application» du traité aux régions ultrapériphériques. Un amendement parlementaire avait proposé d'inclure dans l'article 73 la possibilité pour la loi et le règlement de prendre des «mesures spécifiques ». Cet amendement a été repoussé par le Gouvernement au motif que la notion d'adaptation incluait celle de mesures spécifiques. La différence, selon nous, est que la mesure spécifique ne nécessite pas forcément en amont un texte de portée générale alors que l'adaptation suppose l'existence d'une loi ou d'un règlement de portée générale auquel il est fait des adaptations. Ceci étant, les autorités centrales de l'Etat n'ont aucune obligation de tenir compte des « caractéristiques et contraintes particulières » des territoires concernés. Il est dit dans l'article 73 que les lois et règlements «peuvent» faire l'objet d'adaptations. Tout repose sur la volonté politique du pouvoir central de faire ou de ne pas faire. On constate donc que le constituant français ne s'est pas inscrit explicitement dans l'optique d'un devoir de développement de l'Etat vis-à-vis de territoires ultramarins dont les "caractéristiques et contraintes particulières », pourtant reconnues par lui, appellent des mesures spécifiques ; l'objectif de ces mesures n'étant pas, en soi, de s'éloigner du droit commun, par idéologie différentialiste, mais d'adopter les dispositions les plus appropriées pour favoriser le développement économique, social et culturel des populations concernées.

- Le pouvoir d'adaptation décentralisé ne peut intervenir que dans les matières législatives et parlementaires des DOM-ROM.

- Le pouvoir local d'adaptation ou d'adoption de règles particulières est subordonné à l'adoption par le Parlement d'une loi d'habilitation «dans les conditions et sous les réserves prévues par une loi organique »

Le Parlement garde un pouvoir discrétionnaire d'appréciation de l'opportunité de délivrer ou pas les habilitations demandées par les collectivités territoriales concernées. Le pouvoir local est donc ainsi dépendant de la volonté de la majorité parlementaire du moment.

Il convient de noter que la loi d'habilitation peut faire l'objet d'une saisine du Conseil constitutionnel qui peut ainsi apprécier sa conformité à la Constitution et développer à cette occasion une jurisprudence restrictive. 
limité

- Le champ des possibilités d'adoption des règles particulières est très

Il est limité par l'énumération faite à l'alinéa quatre de l'article 73 et pourra l'être davantage encore par la loi organique qui pourra prévoir des réserves. De plus, ce qui est logique, les habilitations «ne peuvent intervenir lorsque sont en cause les conditions essentielles d'exercice d'une liberté publique ou d'un droit constitutionnellement garanti ». Les règles adoptées demeurent bien entendu des actes administratifs, quoique intervenant dans le domaine de la loi, et susceptibles du contrôle juridictionnel pertinent ${ }^{37}$.

- L'applicabilité de l'alinéa 4 aux collectivités de l'article 74 ne crée pas un amalgame des régimes législatifs

Les lois organiques qui définissent le statut de chaque collectivité régie par l'article 74 ne peuvent transférer de l'Etat vers ces collectivités des compétences portant sur les matières énumérées à l'article 73 alinéa quatre. Le seul fait que l'énumération vaille pour les deux catégories statutaires ne signifie pas qu'il y a confusion entre elles. Le rapprochement se fait ici dans la restriction et non dans l'extension des domaines de compétence. Il apparaît également qu'il y a une différence fondamentale entre les régimes de l'article 73 et de l'article 74 .

En effet, pour les collectivités placées sous le régime de l'article 73, il existe un droit constitutionnel à bénéficier de l'application de plein droit des lois et règlements dans tous les domaines. Le législateur ne peut remettre en question ce droit. Celui-ci n'existe pour les collectivités régies par l'article 74 que pour certaines matières régaliennes. Pour le reste, le législateur organique est libre de doser à sa guise le degré d'identité et de spécialité de chaque collectivité régie par cet article 74.

Dans le cadre de l'article 73, il n'y a pas d'habilitation permanente à réglementer dans le domaine de la loi. Dans le cadre de l'article 74 , la loi organique statutaire attribue à la collectivité un pouvoir permanent de réglementer dans le domaine de la loi concernant certaines matières.

\footnotetext{
${ }^{37}$ Comme le note Yves Brard, «les dérogations envisageables n'apporteront que quelques gouttes de spécialité dans un océan d'identité. Le principe d'assimilation est assoupli, mais il n'est qu'assoupli, assoupli mais pas assoupi, si l'on peut dire », ibidem, p.121.
} 
Les différences de régime apparaissent très nettement quand on compare les dispositions de l'article 73 à celles contenues dans l'article 74 relatives aux collectivités qui sont dotées de l'autonomie (mesures justifiées par les nécessitées locales qui peuvent être prises en faveur de la population des collectivités dotées de l'autonomie, contrôle spécifique du Conseil d'Etat etc.).

Certes, l'avis du Conseil d'Etat formulé sur le projet de loi initial émettait au nombre de ses réserves que "les alinéas 3 et 4 du nouvel article $73 . . . o n t$ paru porter une atteinte excessive au principe d'assimilation législative pourtant réaffirmé $»^{38}$. Mais dans l'arrêt Feler précité, il n'a pas considéré que "ces atteintes excessives » avaient eu pour conséquence un abandon de ce régime. En effet, s'il avait considéré que l'article 73 ne relevait plus de l'identité législative avec possibilité d'adaptations, il aurait conclu que le décret présidentiel, en soulignant que la nouvelle collectivité demeurait régie par le principe de l'identité législative avec possibilité d'adaptations, trompait les électeurs et manquait ainsi de loyauté. Il ne l'a pas fait. Cet arrêt confirme donc implicitement qu' avec l'article 73, nouvelle rédaction, on demeure dans le régime de l'identité législative avec possibilité d'adaptations. Il convient, toutefois de noter que, compte tenu de son avis, selon lequel il considérait que le nouvel article 73 portait «une atteinte excessive » au principe d'assimilation législative, on peut aisément augurer pour l'avenir, de la part du Conseil d'Etat et sans doute aussi du Conseil constitutionnel, d'une interprétation restrictive du champ de l'adaptation et des possibilités de dérogation.

\section{CONCLUSION}

Au total, l'instrumentalisation du droit s'est avérée évidente pendant la campagne précédant la consultation du 7 décembre 2003. Toutefois, cette instrumentalisation fut d'autant plus facile que le vocabulaire est ambigu et que les textes juridiques ne sont pas d'une clarté totale sur un certain nombre de points essentiels. Il en résulte un doute sur le fait de savoir si les électeurs se sont prononcés en toute connaissance de cause le 7 décembre 2003. Ceci ajouté au faible écart enregistré entre le NON et le OUI et au fait que les

${ }^{38}$ Rapport annuel d'activité du Conseil d'Etat, EDCE, 2003, n ${ }^{\circ}$ 54, p.56. 
majorités, aussi bien au Conseil général qu'au Conseil régional, issues des élections de 2004 sont composées de groupes politiques favorables au changement, il semble très probable que le débat sera relancé.

Dans cette hypothèse, il s'avèrerait utile de proposer aux électeurs des choix à partir de concepts clarifiés (notamment par une révision constitutionnelle). Il nous semble indispensable d'être clair sur les notions de changement de catégorie statutaire et de changement de statut, de permettre à ceux qui sont concernés de se prononcer sur le principe mais aussi sur le contenu de la réforme envisagée et de comprendre la portée non seulement juridique mais aussi démocratique, économique, sociale et culturelle des différents choix proposés, autrement dit l'intérêt que peut avoir une réforme institutionnelle pour le développement.

\section{RESUME}

La consultation du 7 décembre 2003 à la Martinique : instrumentalisation politique et ambiguïtés conceptuelles

Cet article propose quelques réflexions rétrospectives sur la consultation du 7 décembre 2003 en Martinique. Après avoir mis l'accent sur les ambiguïtés juridiques qui ont favorisé une instrumentalisation politique, il pose la question d'une nécessaire clarification des concepts et des procédures y compris au moyen d'une révision constitutionnelle, il montre comment la consultation a été faussée par l'attisement de la peur de l'indépendance.

Il souligne l'ambiguïté de la notion de statut départemental qui favorise un discours sur le lien indissociable entre l'organisation administrative départementale et le régime législatif d'application de plein droit avec adaptation. Il insiste sur l'instrumentalisation des notions d'évolution institutionnelle et de changement de statut. Il indique à ce sujet la position du Conseil d'Etat. Il relate les évolutions intervenues au cours de l'élaboration de la révision constitutionnelle promulguée le 28 mars 2003, relatives à la notion de statut, à celle de collectivité à statut particulier et aux procédures de changement.

L'article pose également la question de savoir si certaines impasses ne sont pas inhérentes au texte constitutionnel lui-même (impossibilité de consulter les électeurs sur un projet précis mais sur le seul principe de la substitution d'une collectivité nouvelle aux collectivités départementales et régionales ou du passage du régime de l'article 73 à celui de l'article 74 et vice versa). Il procède à une analyse de la portée et des limites de la nouvelle rédaction de l'article 73 et propose un certain nombre de clarifications, notamment l'utilité de distinguer 
d'une part changement de catégorie statutaire (notamment passage de 73 à 74 et vice versa) et d'autre part changement de statut (plusieurs statuts possibles à l'intérieur de chaque catégorie statutaire). Il souligne le lien nécessaire entre statut et projet de développement.

Mots-clés : articles 73 et 74, catégorie statutaire, changement de statut, Constitution française, consultation populaire, évolution institutionnelle, révision constitutionnelle, statut départemental.

\begin{abstract}
The December 7, 2003 Referendum in Martinique: political instrumentalisation and conceptual ambiguity

This article proposes a few retrospective reflections on the December 7, 2003 referendum in Martinique. Having stressed the legal ambiguities which fostered political instrumentalisation and raised the question of the need for clarifying concepts and procedures, including the re-examination of the French Constitution, it illustrates how the referendum was warped by poking up the fear of independence.

It emphasises the ambiguity of the notion of the status of Departement, inducing dialogue on the indivisible ties existing between the administrative organisation of the Departement and the legislative system of rightful application with adaptation. The article insists upon the instrumentalisation of the notions of institutional change and a change in political status. On this topic, it outlines the position of the French Council of State. It relates the changes occurring during the drafting of the reviewed Constitution promulgated March 28, 2003 concerning the notions of political status, of collective institutions with specific status and of the procedures for change.

The article also raises the question of whether certain deadlocks are not inherent to the Constitutional text per se (the impossibility of a referendum regarding a precise project and not solely on the premise of substituting a new institution to the present General and Regional Councils or passing on from the regime in Article 73 to that of Article 74 and vice versa). It proceeds with an analysis of the scope and the limits of the new drafting of Article 73 and proposes a certain number of clarifications, in particular, the use of distinguishing between a change in status category (especially moving from 73 to 74 and vice versa) and a change in status (several statuses possible within each status category). It highlights the necessary link between status and project for development.

Key Words: Articles 73 and 74, status category, change in status, French Constitution, referendum, institutional change, re-examination of the Constitution, status as a full-fledged Department.
\end{abstract}

